\title{
PFPRINTE

\section{4) Associação entre os níveis séricos de vitamina D e os indicadores de Síndrome Metabólica em idosos}

\author{
Thatiana Siqueira de Freitas Gonçalves ${ }^{1}$; Karina Eraclea Lara Ferreira Parreira'; \\ Joyce de Souza Diniz'; Fabiani Lage Rodrigues Beal²
}

\section{Resumo}

A vitamina D é comumente conhecida por seus efeitos sobre o metabolismo ósseo. Porém, em virtude da presença de receptores específicos dessa vitamina em várias regiões do corpo, os baixos níveis séricos da Vitamina D têm sido associados à diversos tipos de condições patológicas, entre as quais a Síndrome Metabólica. Diversos estudos têm apontado para prevalência da hipovitaminose D e da Síndrome Metabólica em idosos. Dessa forma, o presente trabalho realizou uma revisão sistemática da literatura nas bases de dados LILACS, SciELO e MEDLINE dos anos de 2008 até 2018, para verificar a associação entre essas variáveis, especificamente na população idosa. A análise dos artigos encontrados apontou para uma associação entre a hipovitaminose D e a Síndrome Metabólica quando todos os indicadores dessa síndrome eram analisados em conjunto. Quando os indicadores eram relacionados isoladamente com a hipovitaminose D, essa associação não pôde ser confirmada, suscitando a necessidade de novos estudos nessa área, principalmente entre os idosos.

Palavras-chave: Vitamina D. Síndrome Metabólica. Idosos. Hipovitaminose D.

\section{Introdução}

A vitamina D é bastante conhecida por desempenhar papel fundamental no desenvolvimento e manutenção do tecido ósseo (HEANEY et al, 2008; KIMBALL, 2008). Na maioria dos indivíduos, a obtenção de Vitamina D em sua forma primária e inativa se dá principalmente através da síntese cutânea, por ação dos raios ultravioleta B (UVB); outras fontes dessa vitamina são a alimentação ou o uso de suplementos. Após passar por processos de hidroxilação hepática, a forma ativa da Vitamina D é obtida nos rins por meio de reações enzimáticas (KIMBALL et al, 2008; BARRAL et al, 2007).

Mestrandas no Programa de Pós-Graduação Stricto Sensu em Gerontologia, Universidade Católica de Brasília. Endereço de correspondência: QS 7 - Lote 01 - EPCT - Águas Claras - Brasília, Brasil. Email (na ordem em que aparecem): thatifreitasjf@gmail.com; karinaparreira@yahoo.com.br; joysdiniz@gmail.com

2 Professora no Programa de Pós-Graduação Stricto Sensu em Gerontologia, Universidade Católica de Brasília. Email: fabiani@p.ucb.br 
Inúmeros estudos recentes sugerem que a Vitamina D possui outras funções biológicas, além daquelas relacionadas ao metabolismo ósseo, em virtude da presença de receptores VDR (do inglês Vitamin D receptor) no núcleo de células localizadas em diversas partes do corpo como rins, glândulas paratireoides e intestino (KIMBALL et al, 2008; LIU et al, 2009; BOUILLON et al, 2008; SCHUCH et al, 2009). Dessa forma, os baixos níveis séricos de vitamina $\mathrm{D}$ têm sido associados ao aumento do risco de desenvolvimento de vários tipos de câncer, doenças autoimunes, diabetes tipo 2, obesidade, hipertensão arterial, doenças cardiovasculares e Síndrome Metabólica (CORNACINI et al, 2015; SCHUCH et al, 2009).

A Síndrome Metabólica pode ser definida como um transtorno complexo, caracterizado pela presença de um conjunto de fatores de riscos cardiovasculares associados à deposição de gordura na região abdominal, obesidade visceral, dislipidemia aterogênica, hipertensão, resistência à insulina e outras comorbidades (como esteato-hepatite não alcoólica e apneia obstrutiva do sono) (SOCIEDADE BRASILEIRA DE DIABETES, 2017). Sua prevalência no mundo ainda é bastante variável em virtude da diversidade de critérios existentes para a realização do diagnóstico. Porém, estima-se que um quarto da população mundial tenha essa síndrome, e que ela seja responsável por $7 \%$ da mortalidade global e por $17 \%$ dos óbitos ligados às doenças cardiovasculares (INTERNATIONAL DIABETES FEDERATION, 2006; LIRA NETO et al, 2010; REAVEN, 2010).

Em uma metanálise realizada com latino-americanos por Márquez-Sandoval et al (2011), foi constatado que 24,9\% dos participantes apresentaram a Síndrome Metabólica, sendo ligeiramente mais frequente em mulheres (25,3\%) que em homens (23,3\%). Moreira et al (2014) em um estudo transversal utilizando 1369 brasileiros verificaram a prevalência da Síndrome Metabólica em 22,7\% dos indivíduos, sendo 3 vezes maior a prevalência de Síndrome Metabólica em pacientes idosos - 40,4 \% em pacientes entre 60-69 anos e 42,6\% em pacientes com mais de 70 anos.

De igual modo, verifica-se que existe uma maior prevalência de hipovitaminose D (deficiência: níveis de $25(\mathrm{OH}) \mathrm{D}$ menores que $20 \mathrm{ng} / \mathrm{mL}$; insuficiência: níveis de $25(\mathrm{OH}) \mathrm{D}$ entre 20 e $29 \mathrm{ng} / \mathrm{mL}$ ) em pacientes idosos do que no restante da população; estima-se que $50 \%$ dos idosos no mundo possuem baixos níveis séricos de Vitamina D (ADAMS et al, 2010; HOLICK, 2007; FERREIRA, 2016). No Brasil, a prevalência de hipovitaminose D em idosos varia bastante, principalmente de acordo com a região do país (SOCIEDADE BRASILEIRA DE ENDOCRINOLOGIA E METABOLOGIA, 2014). Diversos estudos regionais ratificam tal afirmação: Saraiva et al (2005) encontraram uma prevalência média de hipovitaminose D em idosos residentes em São Paulo - SP de 57,3\%; em Porto Alegre - RS, Scalco e colaboradores (2008) realizando um estudo com idosos institucionalizados encontraram uma prevalência de 85,7\% de pacientes com hipovitaminose D; Neves et al (2012), através de estudo realizado em João Pessoa - PB, detectaram 37\% dos idosos com hipovitaminose D; no Distrito Federal, 70,9\% dos idosos testados por Galvão et al (2014) eram portadores de hipovitaminose D.

Em virtude dessa prevalência da Síndrome Metabólica e hipovitaminose D em idosos, o presente trabalho teve como objetivo realizar uma revisão sistemática da literatura, verificando em artigos publicados nas bases de dados disponíveis, a associação entre essas duas variáveis nessa parcela da população. 


\section{Material e Métodos}

Foi realizada uma revisão sistemática da literatura utilizando-se as bases de dados LILACS, SciELO e MEDLINE (via PubMed); nas bases LILACS e SciELO a busca foi executada com os descritores "vitamina D" e "síndrome metabólica". Na MEDLINE empregou-se os mesmos descritores, porém traduzidos para a língua inglesa: "vitamin d" e "metabolic syndrome". Para a obtenção de trabalhos que relacionassem ambos os termos utilizou-se o operador booleano "and".

Após a identificação dos artigos, procedeu-se a análise considerando como critérios de inclusão: estudos publicados no período compreendido entre julho de 2008 a julho de 2018; publicações nas línguas português, inglês e espanhol; e estudos com objetivos principais ou secundários de analisar a correlação entre os níveis séricos de vitamina $\mathrm{D}$ e os indicadores de Síndrome Metabólica. Os critérios de exclusão considerados foram: estudos que não envolveram idosos - indivíduos com idade inferior a 60 anos; trabalhos que realizaram revisão da literatura; e publicações de relatos de caso, Anais de Congresso e Abstracts, Respostas ou Comentários de artigos originais.

\section{Resultados}

Foram encontrados um total de 413 artigos; destes 403 foram excluídos por não se enquadrarem nos critérios previamente definidos. Das 10 publicações restantes, apenas 02 tratavam de estudos direcionados à população idosa; os demais artigos encontrados eram de trabalhos realizados com pacientes de várias idades ou direcionados para uma população específica - como mulheres pós-menopausa, mas por envolverem idosos em seus estudos, foram incluídos na presente revisão.

Vitezova et al (2015) analisaram 3240 idosos, com média de idade de 71,2 anos, e concluíram que quanto mais altos eram os níveis séricos de Vitamina D nesta população, menor era a prevalência de Síndrome Metabólica encontrada. Schimitt et al (2018), em estudo realizado com 463 mulheres na pós menopausa - idade entre 45 e 75 anos, detectaram a presença da Síndrome Metabólica em 57,8\% das mulheres idosas com hipovitaminose D, contra 39,8\% naquelas com quantidades suficientes de Vitamina D. Silva et al (2015) verificaram que dos idosos com diagnóstico de hipovitaminose D, 69,2\% eram portadores de Síndrome Metabólica. Damasceno et al (2015) realizaram um estudo com 545 homens, com idade média de 58 anos; constataram que a prevalência de Síndrome metabólica foi maior nos indivíduos idosos com hipovitaminose D comparados com os sujeitos sem hipovitaminose, respectivamente $41,11 \%$ e 28\%. Makariou et al (2012) analisaram 110 indivíduos saudáveis e verificaram que os idosos portadores de Síndrome Metabólica apresentaram níveis significativamente mais baixos de vitamina $\mathrm{D}(11,8 \mathrm{ng} / \mathrm{mL})$ em comparação com os não portadores da síndrome $(17,2 \mathrm{ng} / \mathrm{mL})$.

Outros estudos porém, ao relacionarem individualmente os parâmetros usados para o diagnóstico de Síndrome Metabólica e os níveis séricos de vitamina D, não encontraram relação inversamente proporcional entre essas variáveis, ou detectaram diferenças significativas entre os pacientes com hipovitaminose $\mathrm{D}$ e os com níveis normais dessa vitamina (GRIMNES 
et al, 2011; YIN et al, 2012). Santos (2016) em um estudo realizado com 177 mulheres na fase pós menopausa, não observou relação inversa entre os níveis séricos de Vitamina D e os níveis de pressão arterial sistólicos e diastólicos, tampouco encontrou diferenças significativas quando analisou a glicemia de jejum, obesidade abdominal e perfil lipídico de pacientes com e sem hipovitaminose D. Schnatz et al (2010) ao correlacionarem o HDL com a vitamina D obtiveram uma relação inversamente proporcional, indicando uma tendência de diminuição do HDL ao mesmo tempo em que ocorre o aumento da vitamina D. Andreozzi et al (2016) não conseguiram confirmar a proporcionalidade inversa entre circunferência abdominal e a concentração sanguínea de Vitamina D em um estudo com mulheres na pós menopausa.

\title{
Conclusão
}

Diversos estudos têm apontado uma estreita relação entre os níveis séricos de vitamina D e o aumento no risco de desenvolvimento de Síndrome Metabólica, apesar de poucos deles serem desenvolvidos com os idosos. Os dados encontrados ainda são inconclusivos principalmente em virtude das diversas divergências detectadas e da falta de trabalhos que apontem as relações de causa e efeito entre essas variáveis, suscitando assim a necessidade da realização de novos estudos nessa área.

\section{Association between serum vitamin D levels and Metabolic Syndrome indicators in the elderly}

\begin{abstract}
Vitamin D is commonly known for its effects on bone metabolism. However, due to the presence of specific receptors of this vitamin in several regions of the body, the low serum levels of Vitamin D have been associated with several types of pathological conditions, among them the Metabolic Syndrome. Several studies have pointed to the prevalence of hypovitaminosis D and Metabolic Syndrome in the elderly. Thus, the present study carried out a systematic review of the literature in the LILACS, SciELO and MEDLINE databases from 2008 to 2018 to verify the association between these variables, specifically in the elderly population. The analysis of the articles found pointed to an association between hypovitaminosis D and the Metabolic Syndrome when all indicators of this syndrome were analyzed together. When the indicators were related in isolation to hypovitaminosis $\mathrm{D}$, this association could not be confirmed, provoking the need for new studies in this area, mainly among the elderly.
\end{abstract}

Keywords: Vitamin D. Metabolic Syndrome. Elderly - Hypovitaminosis D.

\section{Referências}

ADAMS J.S.; HEWISON M. Update in Vitamin D. J Clin Endocrinol Metab., v. 95, n. 2, p. 471-478, 2010.

ANDREOZZI, P.; VERRUSIO, W.; VISCOGLIOSI, G.; SUMMA, M.L.; GUELI, N.; CACCIAFESTA, M.; ALBANESE, C.V. Relationship between vitamin $\mathrm{D}$ and body fat distribution evaluated by DXA in postmenopausal women.

Nutrition, v. 3, n. 6, p. 687-692, 2016. 
BARRAL D.; BARROS A.C.; ARAUJO R.P.C. Vitamina D: Uma Abordagem Molecular. Pesq Bras Odontoped Clin Integr, v. 7, n. 3, p. 309-315, 2007.

BOUILLON R.; CARMELIET G.; VERLINDEN L.; VAN ETTEN E.; VERSTUYF A.; LUDERER H.F.; et al. Vitamin D and human health: lessons from vitamin D receptor null mice. Endocrine Reviews, v. 29, n. 6, p. 726-776, 2008.

CORNACINI M.C.; MOSCA L.N.; CLAUDIO C.C.; FUTINO A.S. Níveis séricos de vitamina D3 em mulheres com diabetes mellitus tipo 2. J Health Sci Inst., v. 33, n.1, p. 69-72, 2015.

DAMASCENO M.V.O,; ROSA M.R.B.D.; SOARES A.A.; FERNANDES A.R.G,; BELLUCCI C.H.S.; GOMES C.M., et al. Prevalência de Hipovitamonose D e associação com componentes da Síndrome Metabólica em homens avaliados em programa de detecção do câncer de próstata. Rev. Saúde Col.UEFS, v. 5, n. 1, p. 17-22, 2015.

FERREIRA M.; RIBEIRO R.; MORAES R.; BARGIELA I. O papel da vitamin D na resistência à insulin em idosos. Rev Clin Hosp Prof Dr Fernando Fonseca, v.4, n.1/2, p. 6-9, 2016.

GALVÃO L.O.; REIS C.M.S.; GALVÃO M.F.; MOTTA L.A.C.R. Eficácia da reposição de vitamina d3 em idosos na dose de 50.000 UI por semana durante 8 semanas: é o suficiente? Geriatr Gerontol Aging, v. 10, n. 2, p. 93-100, 2016 .

GRIMNES, G.; FIGENSCHAU, Y.; ALMÅS, B.; JORDE, R. Vitamin D, insulin secretion, sensitivity, and lipids: results from a case-control study and a randomized controlled trial using hyperglycemic clamp technique. Diabetes, v. 60 , n. 11 , p. $2748-2757,2011$.

HEANEY R.P.; ARMAS LAG; SHARY JR.; BELL N.H.; BINKLEY N.;HOLLIS B.W. 25-hydroxilation of vitamin D3: relation to circulating vitamin D3 under various input conditions. The American Journal of Clinical Nutrition, v. 87 , n. 6 , p. $1738-1742,2008$.

HOLICK M.F. Vitamin D deficiency. N Engl J Med., v. 357, p. 266-281, 2007.

INTERNATIONAL DIABETES FEDERATION (IDF). The IDF consensus worldwide definition of the Metabolic Syndrome. IDF Communications, 2006. Disponível em: < https://www.idf.org/our-activities/advocacy-awareness/resources-and-tools/60:idfconsensus-worldwide-definitionof-the-metabolic-syndrome.html>.

KIMBALL S.; FULEIHAN GEL-H.; VIETH R. Vitamin D: a growing perspective. Critical Reviews in Clinical Laboratory SciencesCrit, v. 45, n. 4, p. 339-414, 2008.

LIRA NETO J.C.G.; XAVIER M.A.; BORGES J.W.P.; ARAÚJO M.F.M.; DAMASCENO M.M.C.; FREITAS R.W.J.F. Prevalence of Metabolic Syndrome in individuals with Type 2 Diabetes Mellitus, Rev Bras Enferm, v. 70, n. 2, p. 265-270, 2017.

LIU E.; MEIGS J.B.; PITTAS A.G.; MCKEOWN N.M.; ECONOMOS C.D.; BOOTH S.L.; JACQUES P.F. Plasma 25-hydroxyvitamin $d$ is associated with markers of the insulin resistant phenotype in nondiabetic adults. $\mathbf{J}$ Nutr, v. 139 , n. 2 , p. $329-34,2009$.

MAKARIOU, S.; LIBEROPOULOS, E.; FLORENTIN, M.; LAGOS, K.; GAZI, I.; CHALLA, A.; ELISAF, M. The relationship of vitamin $\mathrm{D}$ with non-traditional risk factors for cardiovascular disease in subjects with metabolic syndrome. Arch. Med. Sci., v. 8, n. 3, p. 437-443, 2012.

MÁRQUEZ-SANDOVAL F, MACEDO-OJEDA G, VIRAMONTES-HÖRNER D, FERNÁNDEZ-BALLART JD, SALAS SALVADÓ J, VIZMANOS B. The prevalence of metabolic syndrome in Latin America: a systematic review. Public Health Nutr., v. 14, n.10, p. 1702-1713, 2011.

NEVES JP, SILVA AS, MORAIS LC, DINIZ ADA S, COSTA MJ, ASCIUTTI LS, et al. 25hydroxyvitamin D concentrations and blood pressure levels in hypertensive elderly patients. Arq Bras Endocrinol Meta bol., v. 56, n. 7, p. 415422,2012 .

REAVEN G.M. The metabolic syndrome: time to get off the merry-go-round? Journal of Internal Medicine, v. 269, p. 127-136, 2010.

SANTOS M.M.A. Associação entre níveis sanguíneos de vitamina d e indicadores da síndrome metabólica em mulheres na pós-menopausa. 2016. (Programa de Pós-Graduação em Alimentos e Nutrição) Universidade Federal do Estado do Rio de Janeiro, RJ. 
SARAIVA G.L.; CENDOROGLO M.S.; RAMOS L.R.; ARAÚJO L.M.Q.; VIEIRA J.G.H.; KUNII I., et al. Influence of ultraviolet radiation on the production of 25 hydroxyvitamin $\mathrm{D}$ in the elderly population in the city of São Paulo (233'S), Brazil. Osteoporos Int, v. 16, n. 12, p. 16491654, 2005.

SCALCO R.; PREMAOR M.O.; FRÖEHLICH P.E.; FURLANETTO T.W. High prevalence of hypovitaminosis D and secondary hyperparathyroidism in elders living in nonprofit homes in South Brazil. Endocrine. v. 33, n. 1, p. 95-100, 2008.

SCHMITT E. B.; NAHAS-NETO J.; BUELONI-DIAS F.; POLONI P.F.; ORSATTI, C. L.; PETRI N.; et al. Vitamin D deficiency is associated with metabolic syndrome in postmenopausal women. Maturitas, v. 107, p. 97-102, 2018.

SCHNATZ, P.F.; NUDY, M.; O'SULLIVAN, D.M.; ETHUN, K.; APPT, S.E.; CLARKSON, T.B. Identification of a mechanism for increased cardiovascular risk among individuals with low vitamin $\mathrm{D}$ concentrations. Menopause, v. 18 , n. 9 , p. $994-1000,2011$.

SCHUCH N.J.; GARCIA V.C.; MARTINI L.A. Vitamina D e doenças en- docrinometabólicas. Arq Bras Endocrinol Metab., v. 53, n. 5, p. 625-33, 2009.

SILVA F.G; RAMOS K.R.L.P.; CALDAS C.P.M; VIEIRA E.M.; CUNHA M.A.L. Avaliação da 25-Hidroxivitamina D em idosos assistidos em uma unidade básica de saúde. Anais V CIEH, v.1, 2015.

SOCIEDADE BRASILEIRA DE DIABETES. Diretrizes da Sociedade Brasileira de Diabetes 2017-2018. São Paulo: Editora Clannad, 2017. Disponível em: < https://www.diabetes.org.br/profissionais/images/2017/diretrizes/diretrizes-sbd-2017-2018.pdf>.

SOCIEDADE BRASILEIRA DE ENDOCRINOLOGIA E METABOLOGIA. Recomendações da Sociedade Brasileira de Endocrinologia e Metabologia (SBEM) para o diagnóstico e tratamento da hipovitaminose D. Arq Bras Endocrinol Metab, v. 58, n.5, p. 411-433, 2014. Disponível em: http://www.pncq.org.br/uploads/2014/qualinews/02_ ABEM585_miolo.pdf

VITEZOVA A.; ZILLIKENS M.C.; VAN HERPT T.W.; SIJBRANDS E.J.; HOFMAN A.; UITTERLINDEN A.G., et al. Vitamin D status and metabolic syndrome in the elderly: the Rotterdam Study. Eur. J. Endocrinol., v. 172, n. 3 , p. $327-335,2015$.

YIN, X.; SUN, Q.; ZHANG, X.; LU, Y.; SUN, C.; CUI, Y.; WANG, S. Serum 25(OH)D is inversely associated with metabolic syndrome risk profile among urban middle-aged Chinese population. Nutr. J., v. 11, n. 68, p. 1-7, 2012. 